\title{
Primary breakup of liquids using a high-speed rotary bell atomizer for spray painting processes
}

\author{
Bo Shen ${ }^{1}$, Q. Ye ${ }^{2}$, O. Tiedje ${ }^{2}$, J. Domnick*1 \\ ${ }^{1}$ University of Applied Sciences Esslingen, Germany \\ ${ }^{2}$ Fraunhofer Institute for Manufacturing Engineering and Automation, \\ Stuttgart, Germany \\ ${ }^{*}$ Corresponding author: joachim.domnick@hs-esslingen.de
}

\begin{abstract}
The present contribution deals with numerical studies of the primary liquid breakup process using a commercial high-speed rotary bell atomizer. The first part of the investigations focuses on the film formation on the distributor disk and the inner surface of the rotary bell. Numerical simulations using the volume of fluid (VOF) approach were carried out. A non-Newtonian liquid is used to investigate the effect of the viscosity on the initial wetting, the film formation process and the film thickness distribution on the bell. The Herschel-Bulkley model is considered to describe the shear-thinning (pseudoplastic) behavior of the fluid. A non-homogeneous film structure is found on the inside surface of the rotary bell. This is also observed in experimental investigations using a high-speed camera.

The following disintegration process is studied by applying an appropriately fine computational mesh. The VOF method and Reynolds stress model are used to calculate the disintegration process and the two-phase flow field. The numerical results are compared with images which are recorded by means of a high-speed camera.
\end{abstract}

\section{Keywords}

Numerical simulation, rotary bell atomizer, film formation, liquid disintegration

\section{Introduction}

High-speed rotary bell atomizers are widely used in automated painting processes. They provide excellent paint film quality as well as high transfer efficiencies due to electrostatic support. Compared to other rotary atomizers for other applications, e.g., spray drying or powder production, the atomizers for painting are characterized by a small bell diameter varying between 20 and $70 \mathrm{~mm}$ and very high rotary speeds of the bell of up to $70,000 \mathrm{~min}^{-1}$.
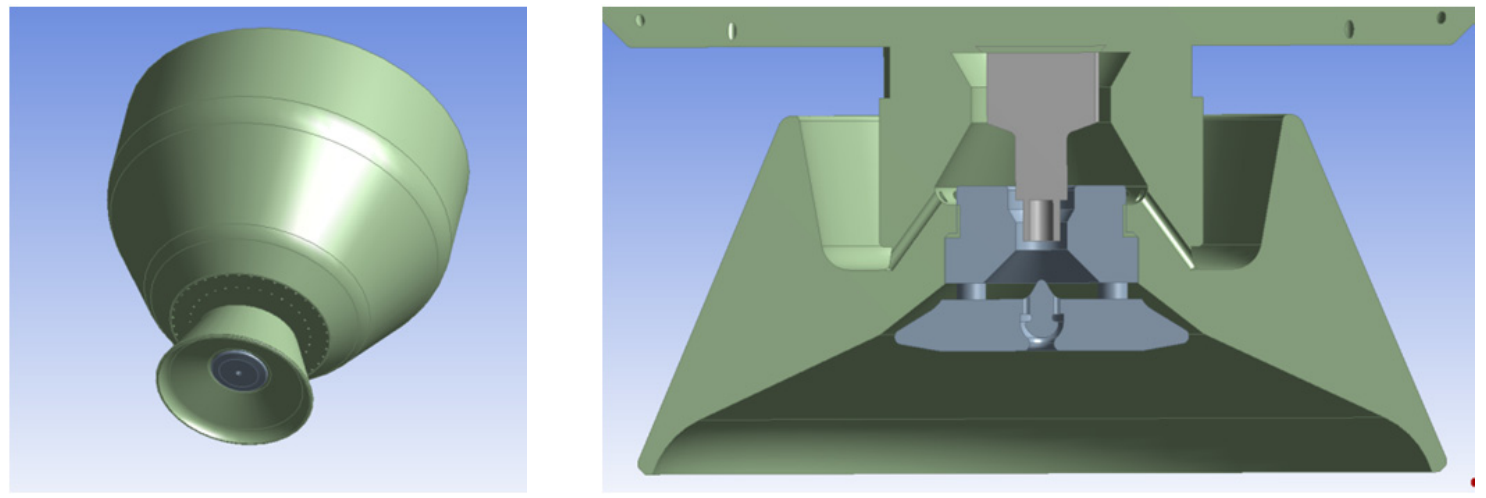

Figure 1. CAD model of the rotary bell atomizer (left) and cross section of the bell (right)

In general, some research works have already been done to investigate the atomization of rotary atomizers (e.g. [1-3]). There are also a few publications presenting results from the experimental works [4] dealing with highspeed rotary bells used in the painting processes. However, the experimental investigations on the film formation on the distributor disk and the bell surface are very difficult due to the complexity of the liquid flow emerging from the nozzle and hitting the surface of the distributor disk. Hence, numerical simulations provide an appropriate alternative. Domnick et al. [5] presented the numerical investigations aiming to calculate the two phase flow field inside the manifold of a rotary bell atomizer and the free film on the bell surface. However, the cell resolution of 
the computational domain was quite limited due to the previously available computational capacity. The viscosity of the paint used in their investigation was chosen to be constant, and the non-Newtonian behavior which real paints can show was not considered. The present contribution summarizes numerical investigations using a significantly finer mesh and a real paint having the shear-thinning behavior. Further, the result of numerical studies on the disintegration of the paint at the bell edge is discussed in this paper.

\section{Characteristics of the atomizer}

The investigations presented in this paper were carried out with a high-speed rotary bell atomizer by EISENMANN LacTec GmbH, which is used in automated paint applications. Figure 1 shows the CAD model of the atomizer and a cross section of the bell. Paint liquid is injected through an orifice which has a narrow diameter of $1.6 \mathrm{~mm}$. This paint supply nozzle is surrounded by an annular gap with a width of $0.5 \mathrm{~mm}$, through which ambient air is sucked down. This gas flow helps to form an initial stable fluid film on the bell and to prevent the formation of ligaments on the bell surface, so that the atomization quality could be improved [6]. Downstream of the paint supply nozzle, there is an inner distributor disk with a diameter of $20 \mathrm{~mm}$. The distributor disk and the bell are assembled together using three bolts. During application they rotate at high speed, while the paint supply nozzle remains static. The paint is injected onto the distributor disk and builds a paint film on it. The paint film is further radially accelerated by centrifugal forces and eventually becomes unstable at a certain distance from the rotating axis. Finally, the paint becomes distributed on the distributor disk surface as well as the inner surface of the bell.

\section{Rheological properties of the paint}

For the investigations shown here, a real paint used in automated painting processes was chosen, whose experimentally measured viscosity is plotted as a function of the shear rate in Figure 2 . The paint shows shearthinning behavior, i.e., the viscosity decreases with increasing shear rate. This kind of non-Newtonian behavior was applied in the simulations shown in this work. The correlation between the viscosity and the shear rate is described by using a power law model, which is known as the Herschel-Bulkley model, and also plotted in Figure 2 .

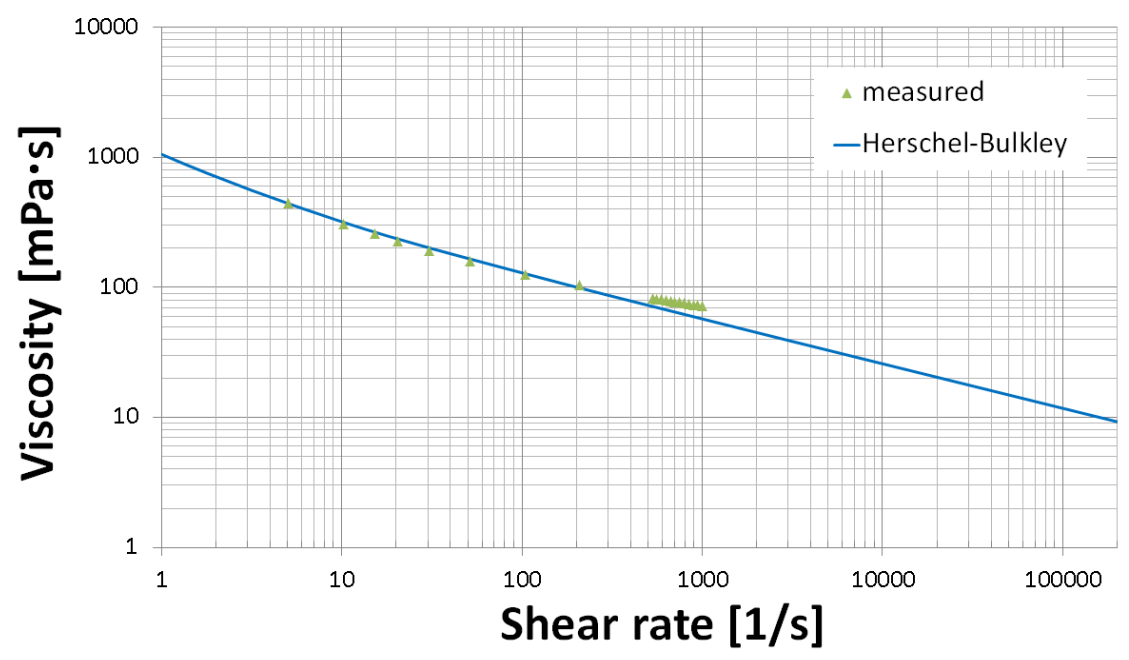

Figure 2. Correlation between viscosity and shear rate of the paint used in the investigations

\section{Numerical method}

A cross-section of the three-dimensional computational domain used in the present investigations is shown in Figure 3. During the generation of the grid, the whole domain was sliced into two parts. Due to the three bolts between the distributor disk and the bell the region above the horizontal surface of the distributor disk is not symmetrical. Thus the middle part of the computational domain had to be built using an unstructured / tetra grid, while in the rest of the domain a structured mesh could be generated. Prism layers were created near the bell as well as near the distributor disk. The height of the first prism layer to the bell was $5 \mu \mathrm{m}$, which provides the necessary resolution for the calculation of the film thickness on the bell. The growth rate of the prism layers is 1.2. The overall mesh cell count was about 8.2 million.

The numerical simulations were carried out using the commercial CFD code FLUENT based on the finite-volume approach. The code was used to solve the unsteady, three-dimensional Reynolds stress transport equations. The 
VOF model was used to calculate the two-phase field and the gas-liquid interface inside the rotary bell. For timedependent VOF calculations, variable time stepping was used in order to automatically change the time step when an interface is moving through dense cells or if the interface velocity is high. The time step was varied in a range between 0.1 and $1 \mu$ s based on the preconfigured maximum global Courant number of 2, which ensures the computational stability of the numerical simulations. The simulations were carried out on a CRAY Cascade Supercomputer at the High-Performance Computing Center of the University of Stuttgart.

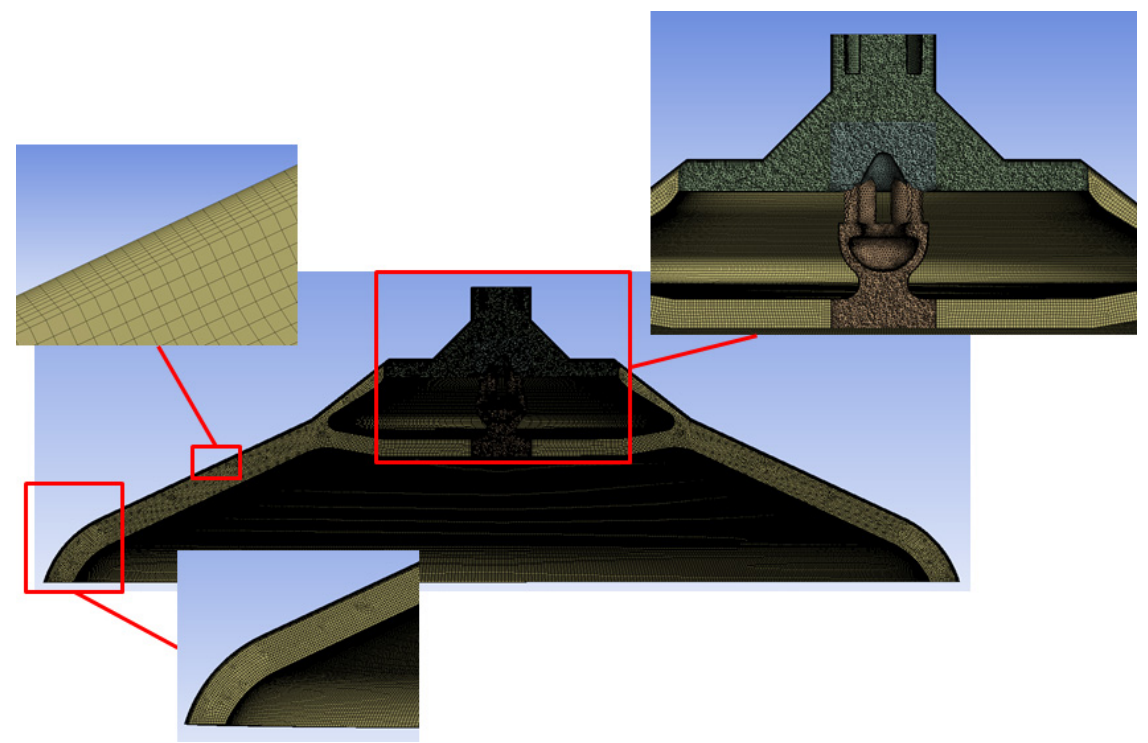

Figure 3. Cross-section of the computational domain

\section{Wetting process on the bell}

The surface wetting on the distributor disk in the quasi-stable state is depicted in Figure 4. The red color indicates that computational cells are completely filled with paint liquid, whereas blue cells contain no paint. The cells in other colors have a volume fraction of paint between 0 and 1 . The left figure shows the case with a paint volume flow rate of $250 \mathrm{ml} / \mathrm{min}$. The horizontal surface of the distributor disk is wetted up to the edge of the three bolts. On the outer inclined surface only several "paint stripes" can be seen. In the case with a larger paint flow rate of $500 \mathrm{ml} / \mathrm{min}$, more areas are wetted. The horizontal surface is almost completely wetted. Due to the rotation of the disk and the relative movement of paint around the bolts, small wake flow regions are generated which are not wetted.
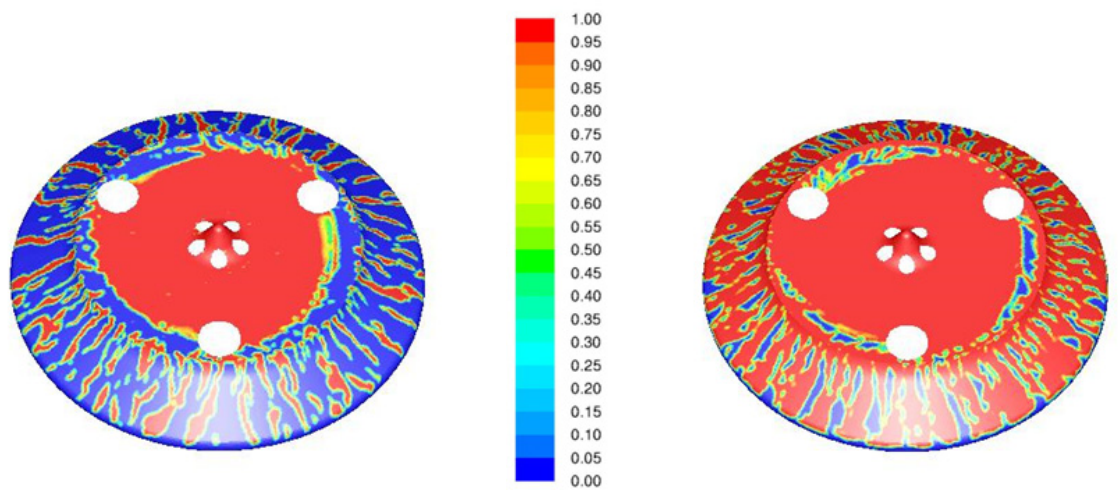

Figure 4. Contour of the paint volume fraction on the distributor disk at a paint volume flow rate of $250 \mathrm{ml} / \mathrm{min}$ (left) and $500 \mathrm{ml} / \mathrm{min}$ (right)

Figure 5 is a snapshot of the contour of the paint volume fraction in the region between the distributor disk and the bell surface from an unsteady simulation, where already a quasi-stable state has been reached. The picture shows the momentary phase distribution in this region. Above the horizontal surface of the distributor disk quite a few drops of liquid can be observed. This indicates that some fraction of the paint material detaches already 
before it reaches the edge of the horizontal surface. This may be caused by a blocking effect of the bolts and the centrifugal force.

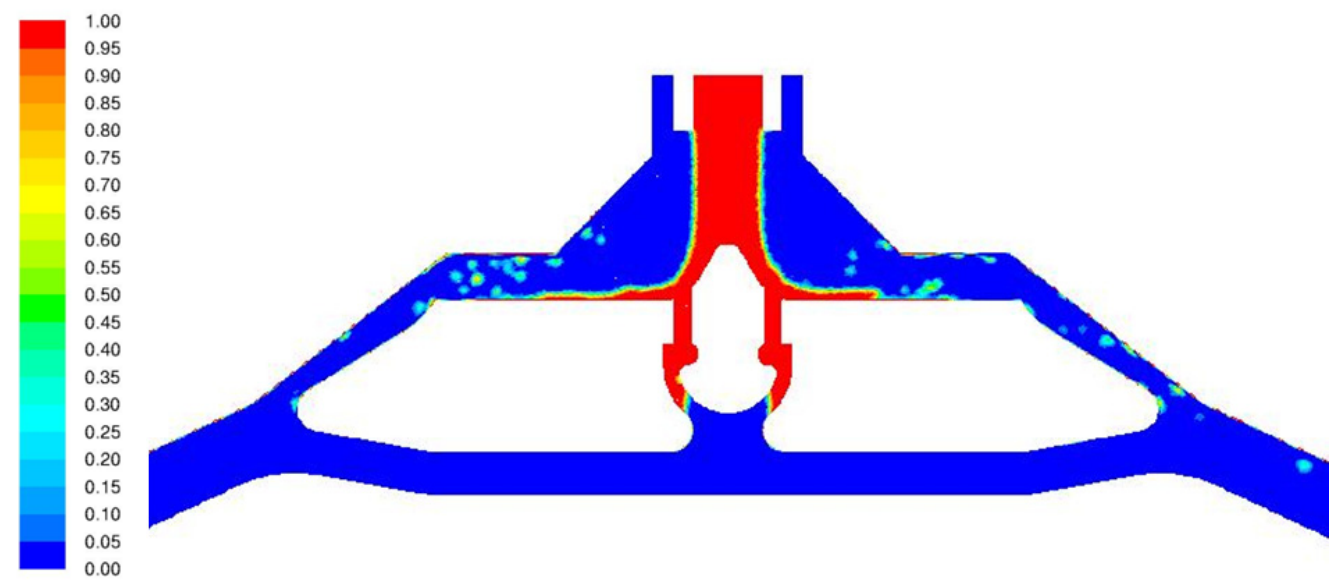

Figure 5. Contour of the paint volume fraction in the region between the distributor disk and the bell surface

The film formation process on the bell surface is shown in Figure 6. In contrast to the distributor disk, the inner surface of the bell is completely wetted after a certain time, e.g., after about $30 \mathrm{~ms}$ for the case with a paint flux of $250 \mathrm{ml} / \mathrm{min}$ and after approx. $20 \mathrm{~ms}$ for $500 \mathrm{ml} / \mathrm{min}$ at the same rotary speed of $40,000 \mathrm{~min}^{-1}$, which is in reasonable agreement with the duration of the wetting process on the bell experimentally measured by means of a high-speed camera.

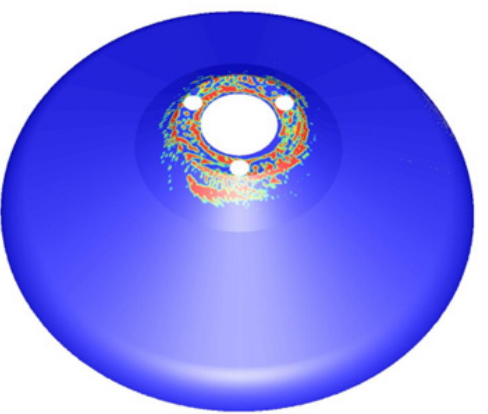

$\mathrm{t}=5.3 \mathrm{~ms}$

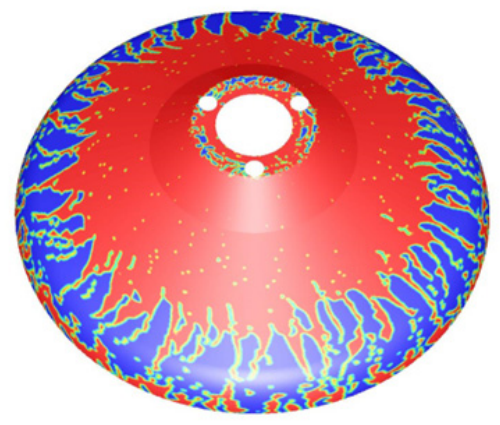

$\mathrm{t}=10.6 \mathrm{~ms}$
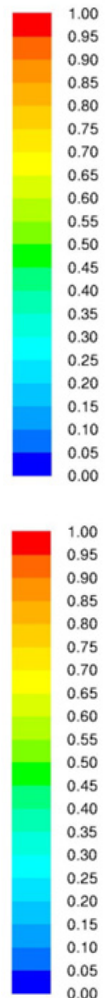

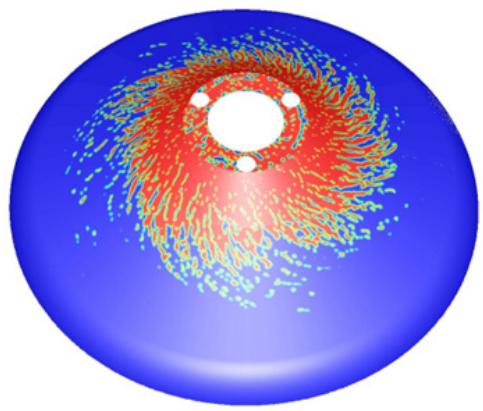

$\mathrm{t}=7.1 \mathrm{~ms}$

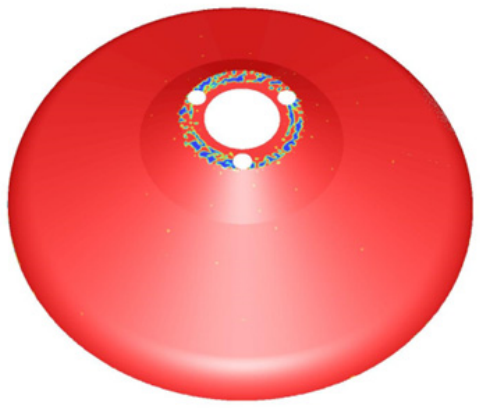

$\mathrm{t}=21.3 \mathrm{~ms}$

Figure 6. Visualization of the film development on the inner bell surface for the case with a paint flux of $500 \mathrm{ml} / \mathrm{min}$ at a rotary speed of $40,000 \mathrm{~min}^{-1}$ 
After the bell surface has been completely wetted, a quasi-stable state is reached. In order to illustrate the paint distribution on the bell surface, an iso-surface positioned at a constant normal distance of $11 \mu \mathrm{m}$ from the inner bell surface was created. The contour of the paint volume fraction on this surface as shown in Figure 7 indicates a non-homogeneous paint distribution. The film thickness in the middle of the bell is relatively high and decreases along the flow direction of the paint. At the edge of the bell the paint film becomes thicker again. In addition, small ripples and a quasi-spiral pattern are obtained on the surface of the paint film. These phenomena can also be observed in experiment using a high-speed camera.
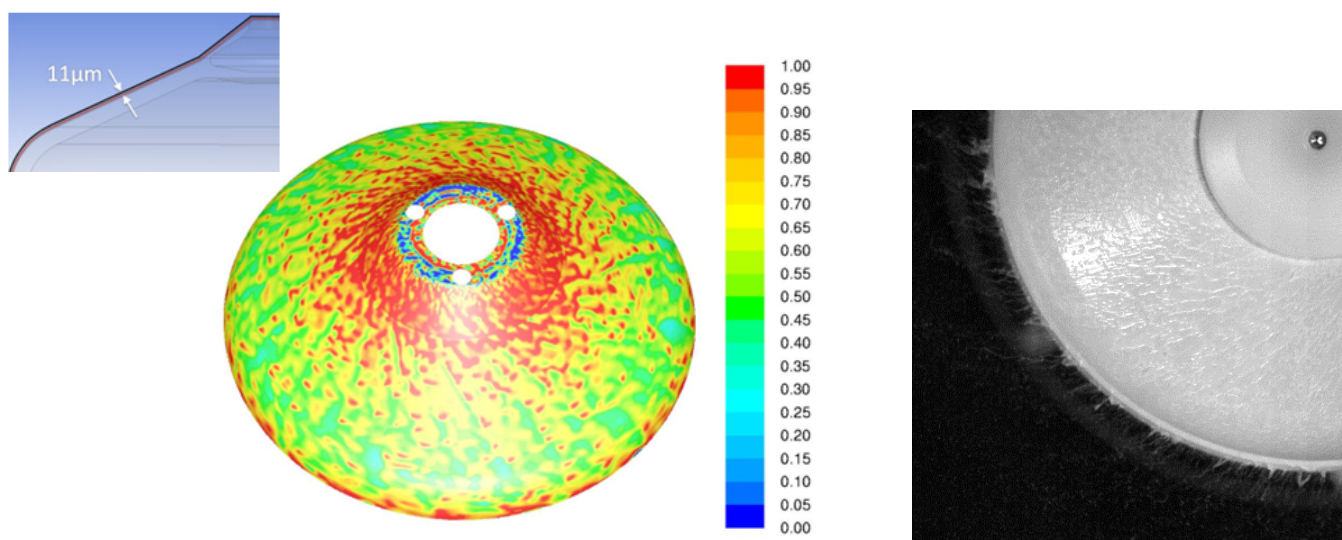

Figure 7. Structure of paint film surface observed in the simulation (left) and in the experiment (right)

A closer look onto the region near the inner bell surface is provided by Figure 8 . The film thickness can be easily obtained from the contour of the volume fraction of the paint. With the thickness of the first prism layer of $5 \mu \mathrm{m}$, the second of $6 \mu \mathrm{m}$, and the third of $7.2 \mu \mathrm{m}$, multiplied by the paint volume fractions in the layers of 1,1 and 0.75 respectively, the thickness of the paint film shown in Figure 8 is calculated to be approximately $16.4 \mu \mathrm{m}$.
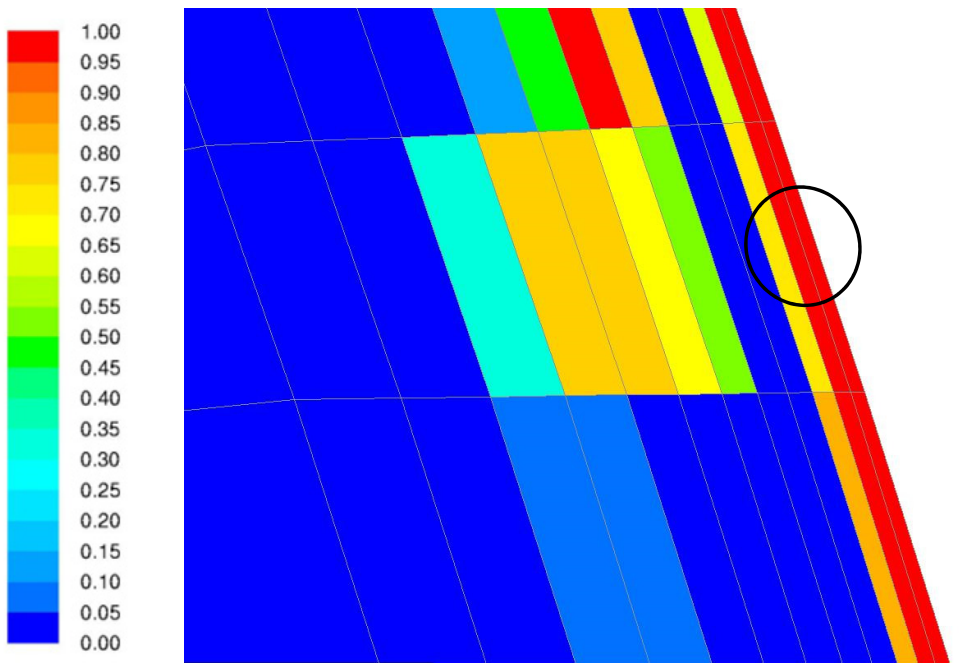

Figure 8. Contour of the paint volume fraction near the inner surface of the bell

Ten measuring points were chosen on the bell surface (Figure 9). The film thickness at each point is calculated using the same method as shown above. The distribution of the film thickness along the outer contour line of the bell is plotted in Figure 9. The tendency shown here is similar to that observed in Figure 7. The film thickness at the bell edge, where subsequent atomization might occur, is determined to be $15 \mu \mathrm{m}$ for the case with a paint flux of $250 \mathrm{ml} / \mathrm{min}$ at the rotary speed of $40,000 \mathrm{~min}^{-1}$. 


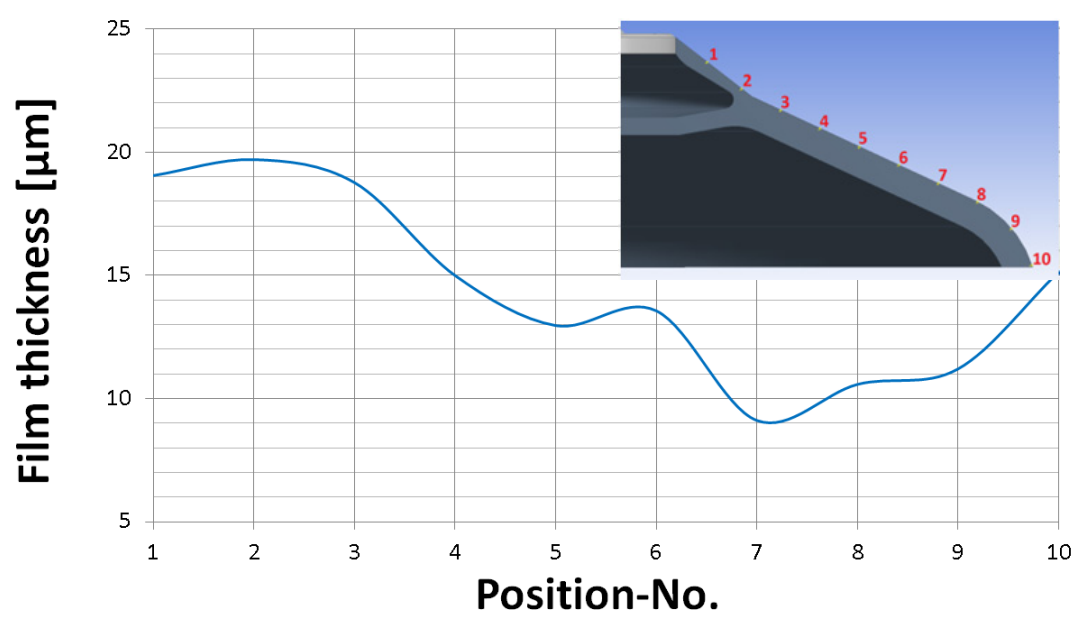

Figure 9. Distribution of film thickness along the flowing direction of the paint

\section{Disintegration process at the bell edge}

Numerical studies on the atomization at the bell edge need a significantly finer mesh in this region. The computational domain as shown in Figure 10 contains only 1/8 of the bell without bolts. The wall boundary of the bell is extended to the fillet on the outer bell surface. The domain is enlarged laterally by $4 \mathrm{~mm}$ and downwards by $2 \mathrm{~mm}$. The total of cell elements in the domain amounts to 17.5 million.
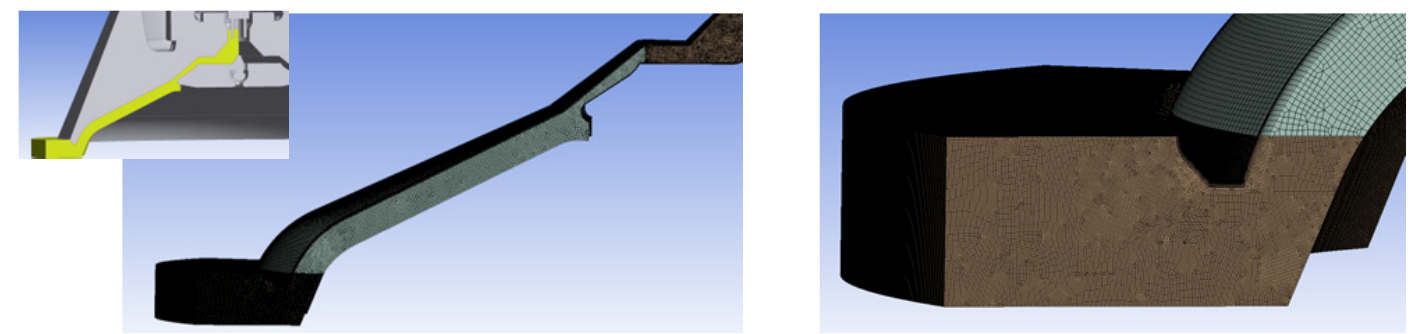

Figure 10. Computational domain for the simulation of atomization

In the simulation for the case with a paint flow rate of $500 \mathrm{ml} / \mathrm{min}$ at a rotary speed of $60,000 \mathrm{~min}^{-1}$, turbulent disintegration is found, because no liquid jet emerging from the bell edge can be observed. This coincides well with previous experimental work by Domnick et al. [4], in which an equation defining the separation between jet and transition disintegration at the rotary bell edge was derived. It is also observed in the simulation that the major paint fraction leaves the bell not directly at the bottom edge of the bell. The paint flows over the bottom surface of the bell, builds a film on the surfaces and reaches the lower edge of the fillet on the outer bell surface, where drops and ligaments are obtained as the paint detaches the bell. This phenomenon can also be observed by means of a high-speed camera.
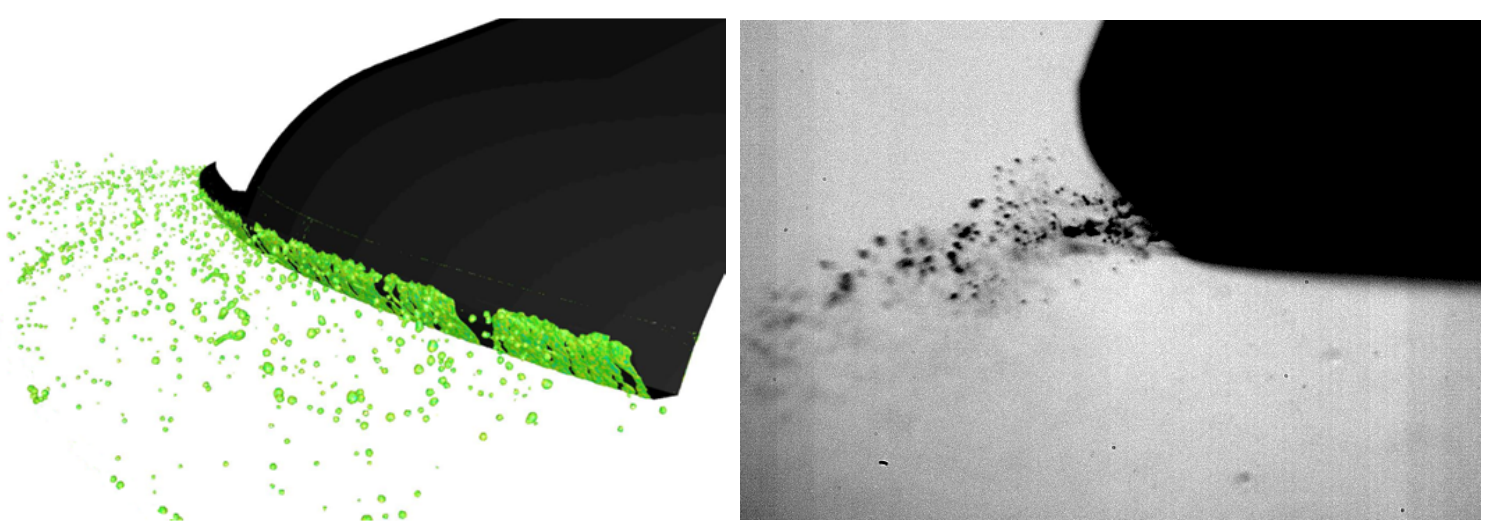

Figure 11. Liquid disintegration at the bell edge observed in the simulation (left) and in the experiment (right) 


\section{Conclusions}

As presented in this contribution, numerical simulations based on the VOF method and Reynolds stress model were carried out to investigate the film formation process of the paint on the distributor disk and the bell itself. The propagating process of the paint from the disk to the bell could be observed. The blocking effect of the bolts, with which the distributor disk and the bell are assembled together, plays an important role in this process. The paint leaves the distributor disk in form of drops or ligaments, reaches the inner surface of the bell and builds a film on it. The paint flows further along the inner surface towards the bottom edge of the bell. After a certain time, the inner surface of the bell is completely wetted by the paint. However, the distribution of the film thickness is not homogeneous. The major part of the paint material does not leave the bell at the bottom edge of the inner bell. Instead, the paint flows further over the bottom surface of the bell in form of a film and reaches the fillet on the outer surface of the bell. There, the paint disintegrates into a chain of drops or ligaments.

For the further atomization study, numerical simulation will be carried out using finer mesh around the bell edge. The computational domain will be limited quite close to the bell edge, in order to save the computational consumption. The corresponding boundary conditions, such as velocity and film thickness distributions, can be obtained from the present numerical simulation results of the film formation on the bell.

\section{Acknowledgements}

The present investigations were supported by AiF Arbeitsgemeinschaft industrieller Forschungsvereinigungen. We thank our colleagues from the Fraunhofer Institute for Manufacturing Engineering and Automation in Stuttgart who provided the results from the experimental work that greatly assisted the present research. We also thank Anyang Wang for his assistance in producing the results used in this paper.

\section{References}

[1] Lefebvre, A. H., 1989, "Liquid Atomization."

[2] Walzel, P., Zerstäuben von Flüssigkeiten, 1990, Chemie Ingenieur Technik, vol. 62, pp. S983-S994

[3] Bayvel, L., Orzechowski, Z., 1993, "Liquid Atomization."

[4] Domnick, J., Thieme, M., 2006, Atomization and Sprays, vol. 16, pp. 857-874

[5] Domnick, J., Yang, Z., Ye, Q., Sep. 8.-10. 2008, 22nd European Conference on Liquid Atomization and Spray Systems

[6] Mehrhardt, E., 1978, Ph.D. thesis, Technical University of Berlin 